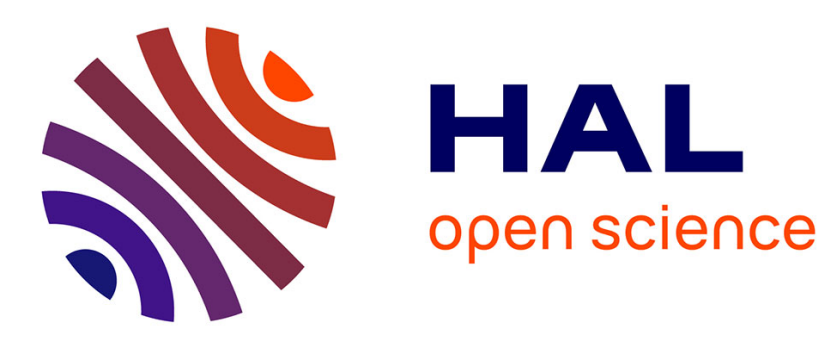

\title{
Automatic Fall Detection System with a RGB-D Camera using a Hidden Markov Model
}

\author{
Amandine Dubois, François Charpillet
}

\section{To cite this version:}

Amandine Dubois, François Charpillet. Automatic Fall Detection System with a RGB-D Camera using a Hidden Markov Model. ICOST - 11th International Conference On Smart homes and health Telematics - 2013, Jun 2013, Singapore, Singapore. pp.259-266, 10.1007/978-3-642-39470-6_33 . hal00914345

\section{HAL Id: hal-00914345 \\ https://hal.inria.fr/hal-00914345}

Submitted on 5 Dec 2013

HAL is a multi-disciplinary open access archive for the deposit and dissemination of scientific research documents, whether they are published or not. The documents may come from teaching and research institutions in France or abroad, or from public or private research centers.
L'archive ouverte pluridisciplinaire HAL, est destinée au dépôt et à la diffusion de documents scientifiques de niveau recherche, publiés ou non, émanant des établissements d'enseignement et de recherche français ou étrangers, des laboratoires publics ou privés. 


\title{
Automatic Fall Detection System with a RGB-D Camera using a Hidden Markov Model
}

\author{
Amandine Dubois and François Charpillet \\ Inria and Université de Lorraine, \\ LORIA, UMR 7503, Vandœuvre-lès-Nancy CEDEX, F-54506, France \\ firstname. lastname@loria.fr
}

\begin{abstract}
Falls in the elderly is a major public health problem because of their frequency and their medical and social consequences. New smart assistive technologies and Health Telematics make it possible to provide elderly with more security and well being at home. A smart home can automatically monitor home activities for early warning in health changes or detecting dangerous situations. One of our objectives is to design an automatic system to detect fall at home, which in its final version will be made up of a network of RGB-D sensors. In this paper, we present a simple and robust method based on the identification and tracking of the center of mass of people evolving in an indoor environment. Using a simple Hidden Markov Model whose observations are the position of the center of mass, its velocity and the general shape of the body, we can surprisingly monitor the activity of a person with high accuracy and thus detect falls with very good accuracy without false positives. An experimental study, that is reported here, has been driven in our smart apartment lab. 26 subjects were asked to perform a predefined scenario in which they realized a set of eight postures. 2 hours of video (216 000 frames) were recorded for the evaluation, half of it being used for the training of the model. The system detected the falls without false positives. This result encourages us to use this system in real situation for a better study of its efficiency.
\end{abstract}

\section{Introduction}

According to a French statistic agency (INSEE), 1200000 people, in France, will be dependent in 2040 against 800000 today. That obviously presents a problem of funding, as most of dependent elderly people are moving into long-term care units. The development of new technologies such as those provided by Robotics or Ambient Intelligent researches could offer an alternative allowing elderly people with loss of autonomy to stay longer at home than they do today by providing them with efficient assistance, especially when they live alone at home.

"Smart houses" for assisting and telemonitoring elderly people at home is not a new idea. In France, in the end of 90ths, TIMC-IMAG laboratory (Grenoble) [1] proposed the concept of HIS (Health Smart Home) and LAAS laboratory in Toulouse [2] launched at the same time the "PROSAFE" project. More recently, 
we can quote the Gerome project in Nice or the Adream project by LAAS. Inria, launched too, two years ago a national program called Pal ("Personal assisting living") in which our group is implied. Several Intelligent home laboratories have been built within this project especially in Nancy where our group stays. A large number of projects about smart houses are ongoing all around the world dedicated either to the management of energy and well being of people or on the development of new services for elderly people. Let us quote "MavHome", "House of Matilda", "Gator Tech Smart House", "Aware Home Research", "Easyliving", "Sensorized Environment for LiFe", "Intelligent Sweet Home".

Fall detection is a service, which is often mentioned by researchers in this domain, elderly people associations, and caregiver. Elderly fall is one of the major health issues affecting elderly people, especially at home. The importance of this problem is shown by the figures of the INPES (2005), which reveal that one elderly people out of three falls in the year. The consequences in term of autonomy for a person experimenting a fall are often dramatic and furthermore increase her risk of mortality. It's not rare that people stay all night on the ground after a fall before someone could assist them. The falls can have serious physical consequences (for example fractures), and also psychological ones (fear of falling again). Generally fallers face far greater problems because they carry out less daily life activities which increases their fragility.

Mainly, two categories of systems exist to detect falls. The first category consists in systems with sensors, that the person wears on her, as the accelerometers, the gyroscopes or the goniometers ([3], [4]). In this category we can add the systems with alarm button where the person must press herself on a button to alert after the fall. The problem of this category is that the person may sometimes forget to wear it. The second category of systems detecting falls is based on environmental sensors. Among them, video camera have greatly attracted the research community. This article is related to this last category of ambiant sensor approach, a RGB-D camera (Kinect) is chosen to detect the falls because it's a low-cost system which may be used by day and by night.

\section{Proposed Approach}

Several methods have been developed so far to detect falls from video cameras. Some authors build a blob around the person [5]. Others track an element of the person's body as the head [6], the head and the feet [7] or human centroid [8]. Finally, the methods to detect falls may be based on the detection of a person near the ground [8], the vertical speed [9] or the scene training of inactivity places [10]. We propose to fusion 3 simple indicators with a Hidden Markov Model (HMM) for filtering RGB-D camera images in order to detect falls.

\section{$2.1 \quad$ Feature extraction}

In this paper, we argue that three simple features are sufficient for detecting falls and discriminating them from other similar situations like sitting or squatting. As presented in Figure 1, we extract the background from depth camera images 


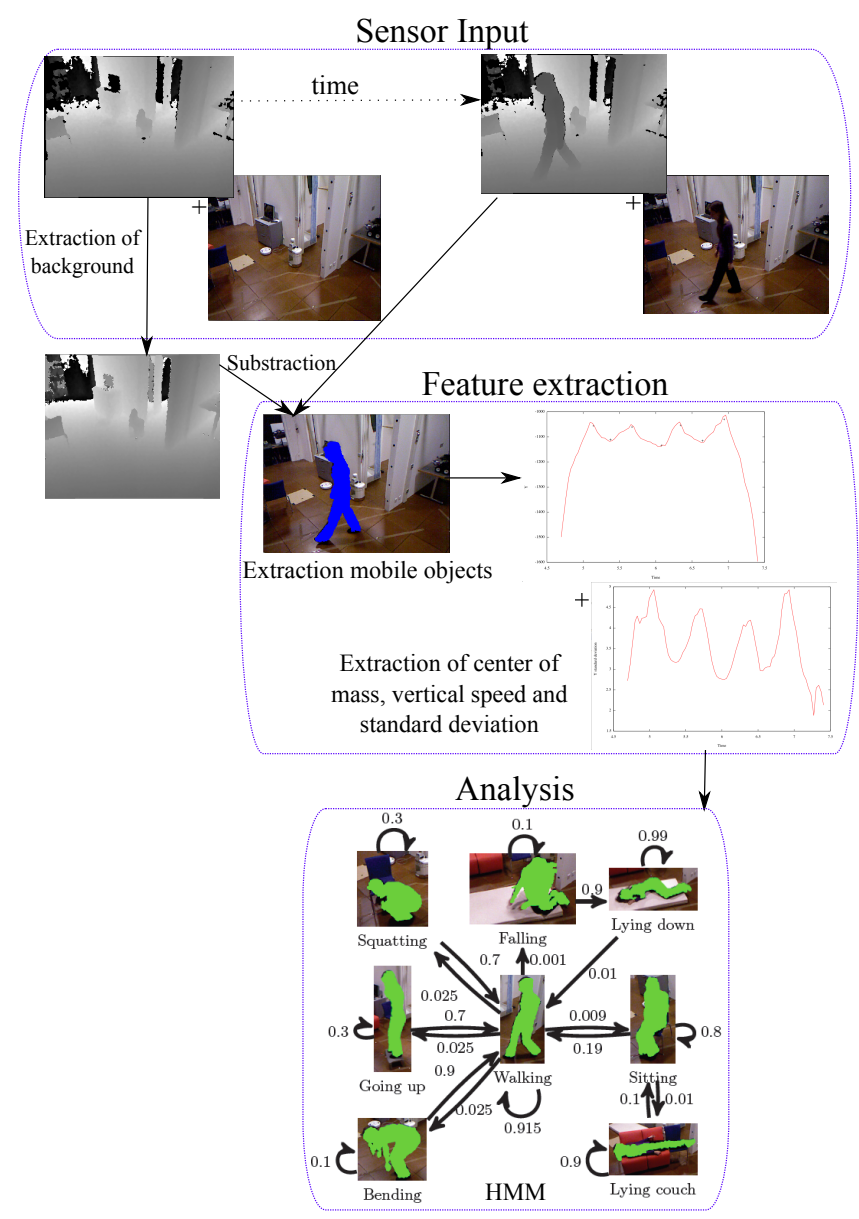

Fig. 1. Fall detection method.

to obtain the mobile points and calculate the position of the center of mass, the vertical speed and the standard deviation of the mobile points.

Extraction of background Several methods can be used for detecting mobile elements in the scene like Gaussian Mixture Model [11], occupancy grid [12]. In this article we use a simple and fast method which consists in averaging the depth map for learning the background [11]. At each time we substract the background distances from the current distances to keep only mobile points. The real world coordinate system is obtained by using the Kinect factory optical parameters and we compensate the camera tilt angle (read from the camera accelerometer sensor) by a rotation on $\mathrm{X}$-axis. We use OpenNI for Kinect to real world transformations. 
Tracking a person and her center of mass The aim is to gather the mobile points (2D pixels) belonging to the same object so as to be able to distinguish several persons in the scene. To gather the mobile points the method "Component labelling" [13] is used. Then we extract the features. The center of mass of the person is calculated as the average location of all the mobile points. The vertical speed is calculated from the position of the center of mass. And the vertical standard deviation is extracted from the point cloud of the person. The image entitled "Extraction mobile objects" of Figure 1 shows the result obtained with the running average and component labelling algorithms. All the mobile points belonging to the same person are gathered in color. The first image entitled "Extraction of center of mass, vertical speed and standard deviation" is the displacement of center of mass of a person on the vertical plane. In this figure, each point represents the center of mass in time. This curve is filtered with the Kalman method [14]. The second curve is the variation of the standard deviation in time.

\subsection{Recognition of the activity of a person}

In this part we present the method used to recognize the activity of a person more particularly to detect the falls. Our method is based on a Hidden Markov Model.

The aim is to create a model allowing to distinguish the falls from other activities. To define these other activities we were inspired by the article of Noury et al [15] where he described scenarios for the evaluation of fall detectors. We retain seven activities (other that falling), postures that a person can take: walking (including the position upright), lying (on a bed, on a couch for example), sitting, lying down, squatting, going up on an obstacle (a chair, a footboard for example) and bending. These eight postures are represented by the eight states of our HMM. The representation of this HMM is shown in Figure 1 in the block "Analysis". The meaning of each state is represented by the picture.

The model and these probabilities were deduced intuitively respecting the following assumptions:

- the most probable states are "walking" and "sitting", for a people at home, and the other states have a small probability in comparison;

- the difference between the state "lying couch" and the state "lying down" is the preceding state. In the case of lying on the ground, the preceding state is "falling" and the probability of this state is small. In the case of lying on a couch the preceding state is "sitting" because we sit down before lying on a couch.

In our HMM, the observations are the three features extracted as described in Section 2.1: the vertical position of the center of mass, the vertical speed and the standard deviation of all the points belonging to the person. The observation function follows a multidimensional normal law whose parameters are calculated from the data of 16 subjects visiting the eight states of the HMM. More explanations on this experiment are given in Section 3. 
To calculate the probability to be in one of the eight states of HMM, we implemented the Forward-Backward algorithm and to calculate the best path we implemented Viterbi [14]. These two algorithms are compared in section 3.2. We assign the same initial probability to each state $(1 / 8)$. We make the hypothesis that it's possible to be in each state at the beginning of the analysis.

\section{Results}

To test our algorithm an experiment is made with 26 subjects. These tests were made in a smart room. Only one camera is placed on the corner as shown in Figure 2. Each subject perform eight situations corresponding to the eight states of the HMM. In Figure 1 concerning the HMM we can see eight pictures corresponding to what was requested from the subjects. To learn the observation function as shown in Section 2.2, the data of 16 subjects are used and the validity of the HMM is tested with the data of 10 other subjects. We test and compare the two algorithms, Forward-Backward and Viterbi.

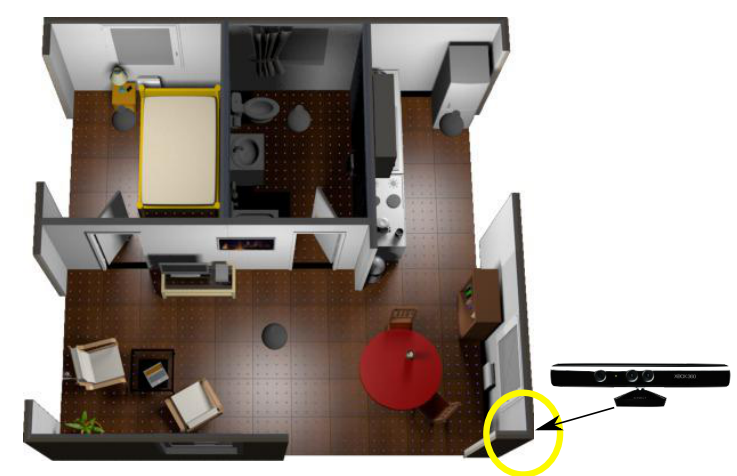

Fig. 2. The smart room and the position of the camera surrounded in yellow.

\subsection{Observation function parameters}

As described in Section 2.2 we learn the mean and covariance matrix of the feature vector composed of the vertical position of the center of mass, of the vertical speed and of the standard deviation of all the points belonging to the person for each situation performed by the 16 subjects. The results are shown in Table 1. The state "going up" and "walking" have a high vertical position whereas "lying down" has a small vertical position. The state "falling" distinguishes itself from the other states by the vertical speed. And concerning the standard deviation there is a difference between "walking" and "going up" on the one hand and "lying down" and "lying couch" on the other hand.

\subsection{Result with Forward-Backward and Viterbi}

We have tested in which state was classified each situation of the 10 subjects not belonging to the learning group by using the Forward-Backward algorithm and 


\begin{tabular}{|c|c|c|c|}
\cline { 2 - 4 } \multicolumn{1}{c|}{} & Vertical position & Vertical speed & Standard deviation \\
\hline Walking & -1163 & 52 & 372 \\
\hline Bending & -1299 & -41 & 308 \\
\hline Squatting & -1424 & 4 & 240 \\
\hline Going up & -975 & 117 & 333 \\
\hline Falling & -1505 & -352 & 216 \\
\hline Lying down & -1871 & -6 & 102 \\
\hline Sitting & -1370 & -10 & 303 \\
\hline Lying couch & -1569 & -3 & 210 \\
\hline
\end{tabular}

Table 1. Mean of the gaussian for each state of the HMM.

the Viterbi algorithm. The two algorithms give the same classification results. For sitting, squatting, lying on a couch, lying down, walking and going up the sensitivity (capacity to detect a state when it is present) is $100 \%$. On the other hand for the 10 situations where each subject is bending the algorithm replaced by state "sitting" or by the state "squatting". The problem is that the observation doesn't allow to dissociate this state enough from the states "sitting" and "squatting", even visually. Concerning the classification of falls, one fall was not detected. This non detected fall corresponds to a subject who rose immediately after he fell, the fall itself being mitigated so the fall was not realistic. This subject doesn't pass in the state "lying down". However, according the HMM that we have defined in section 2.2 , to be in the state "falling" it's necessary to pass in the state "lying down". For falls we can add that the specificity (the capacity of the system to detect the absence of a state when it doesn't appear) is $100 \%$ i.e. there are no false positives.

According to the HMM a person can fall only after the state "walking" which doesn't correspond to reality. We want to know if our algorithm is really robust for detecting the falls. We asked a subject to sit and fall from his chair. The algorithm is robust because it detects the fall even if there are no transition between the state "sitting" and the state "falling" in the HMM.

A last notice, we didn't see differences between Forward-Backward and Viterbi algorithm exept the "transition state". Viterbi give more state for a same situation. We asked a subject to sit and go up on a chair without walking between these two situations. We analysed the results with Forward-Backward algorithm and Viterbi algorithm, as presented in Figure 3. We can notice that Viterbi provided more states. To pass from sitting to going up the subject didn't walk as correctly inferred by Forward-Backward in Figure 3(a) but Viterbi inferred the state walking. It's not correct but Viterbi stick at the contraints provided by the HMM. In the HMM we have proposed that after the state sitting we have the state walking before passing to the other state. Thus in figure 3(b) Viterbi pass by the state walking even if the subject doesn't walk. 


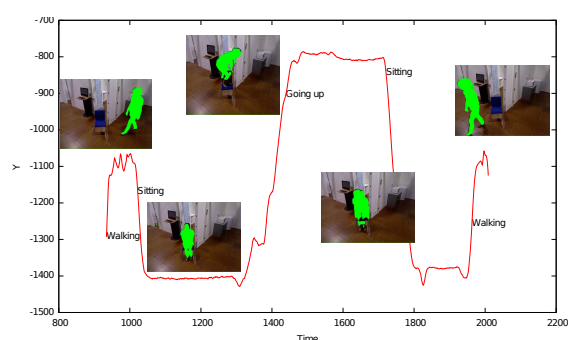

(a) Analysis with Forward-Backward algorithm.

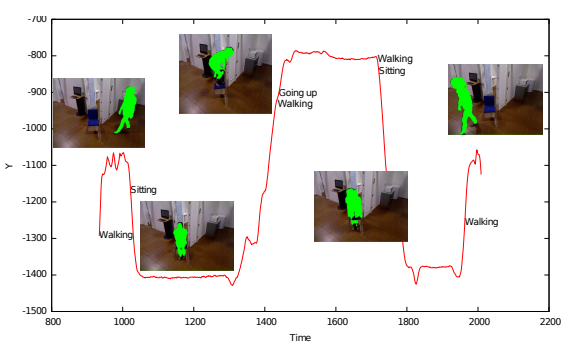

(b) Analysis with Viterbi algorithm.

Fig. 3. Difference between Forward-Backward and Viterbi algorithm.

\subsection{Result in real time and discussion}

In introduction we said that we wanted to develop a system for detecting falls. The results show that our algorithm allows a correct identification of the falls. But these results were obtained by analyzing video sequences. We implemented our algorithm in real time to launch the analysis at the same time as the person is viewed by the camera. Figure 4 shows a person walking and falling. In the top left-hand corner of this figure the image is the depth background learnt and on the right is the current depth image of the camera. In the bottom left-hand corner is the current RGB image with the object detected as foreground drawn in color. And in the bottom right-hand corner the center of mass on the vertical plane is drawn and the algorithm deduced the state in which the person is. We can see in this figure that the algorithm detects correctly the walk and the fall of the person. We can imagine a system which would allow to send a signal to a physician or to a member of the family when it detects that the person has fallen as in Figure 4(b). This system uses a computer and low-cost cameras. Another advantage is that this system allows to preserve the privacy of the person since it works with extracted features and the images don't need to be stored.

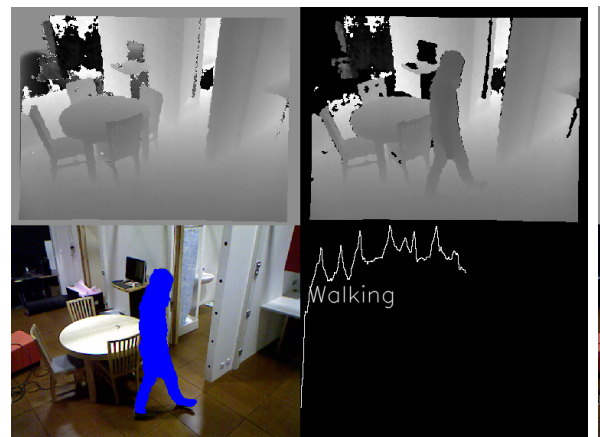

(a) Detection of the walking.

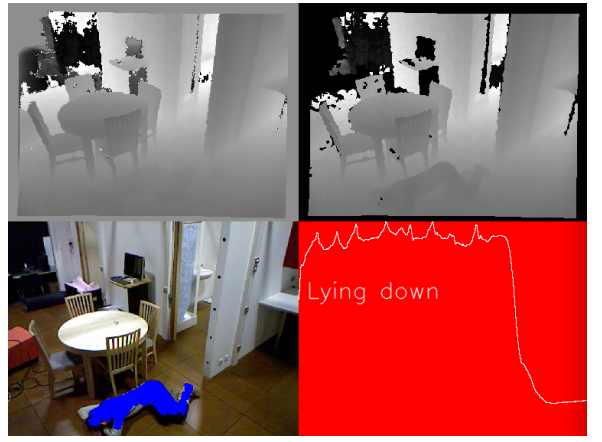

(b) Detection of the fall.

Fig. 4. Real time fall detection. 


\section{Conclusion}

In this paper we presented a system based on RGD-D camera able to detect falls of elderly people while preserving their private life. Our algorithm tracks the center of mass of the person. To know if the person falls we create a HMM with eight states corresponding to eight situations of daily life. The results show that our model distinguishes correctly the falls from the others activities (as sitting, lying on a couch...). The experiment was made on healthy subject and without occlusions. In the future we plan to explore these two problems.

\section{References}

1. Rialle, V., Duchene, F., Noury, N., Bajolle, L., Demongeot, J.: Health" smart" home: information technology for patients at home. Telemedicine Journal and E-Health 8(4) (2002) 395-409

2. Chan, M., Hariton, C., Ringeard, P., Campo, E.: Smart house automation system for the elderly and the disabled. In: Systems, Man and Cybernetics. Intelligent Systems for the 21st Century., IEEE International Conference on. Volume 2. (1995)

3. Bourke, A.K., O'Brien, J.V., Lyons, G.M.: Evaluation of a threshold-based tri-axial accelerometer fall detection algorithm. Gait \& posture 26(2) (2007) 194-9

4. $\mathrm{Wu}, \mathrm{G} .:$ Distinguishing fall activities from normal activities by velocity characteristics. Journal of biomechanics 33(11) (2000) 1497-500

5. Jansen, B., Temmermans, F., Deklerck, R.: 3D human pose recognition for home monitoring of elderly. IEEE Engineering in Medicine and Biology Society. Conference (January 2007) 4049-51

6. Rougier, C., Meunier, J.: 3D Head Trajectory Using a Single Camera. Engineering In Medicine And Biology (2010) 505-512

7. Lyon, U.D.: Méthode robuste pour la détection de chute dans un environnement. Computer (2012)

8. Rougier, C., Auvinet, E., Rousseau, J., Mignotte, M., Meunier, J.: from Depth Map Video Sequences. (2011) 121-128

9. Rougier, C., Meunier, J., St-Arnaud, A., Rousseau, J.: Monocular 3D head tracking to detect falls of elderly people. IEEE EMBC 1 (2006) 6384-7

10. Nait-Charif, H., McKenna, S.: Activity summarisation and fall detection in a supportive home environment. Proceedings of the 17th International Conference on Pattern Recognition, 2004. ICPR 2004. (2004) 323-326 Vol.4

11. Gómez-Conde, I.: Simple Human Gesture Detection and Recognition Using a Feature Vector and a Real-Time Histogram Based Algorithm. Journal of Signal and Information Processing 02(04) (2011) 279-286

12. Dubois, A., Dib, A., Charpillet, F.: Using HMMs for Discriminating Mobile from Static Objects in a 3D Occupancy Grid. IEEE 23rd ICTAI (2011) 170-176

13. Suzuki, K., Horiba, I., Sugie, N.: Linear-time connected-component labeling based on sequential local operations. Computer Vision and Image Understanding 89(1) (January 2003) 1-23

14. Rabiner, L.R.: A tutorial on hidden Markov models and selected applications in speech recognition. In: Proceedings of the IEEE. (1989) 257-286

15. Noury, N., Fleury, a., Rumeau, P., Bourke, a.K., Laighin, G.O., Rialle, V., Lundy, J.E.: Fall detection-principles and methods. Conference proceedings : Annual International Conference of the IEEE Engineering in Medicine and Biology Society. (January 2007) 1663-6 